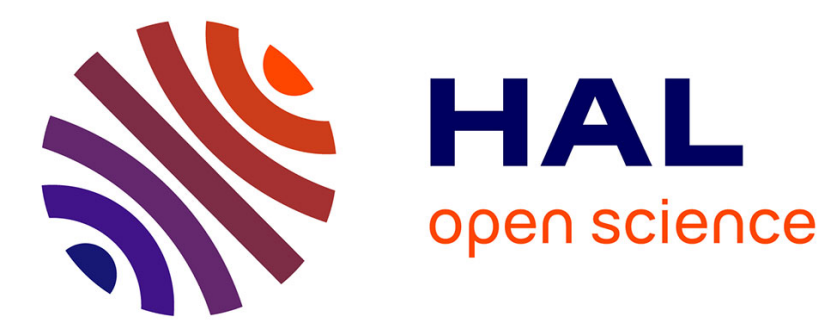

\title{
DEPOSITION OF BORON NITRIDE BY PLASMA ENHANCED CVD USING BORANE AMINE
}

\author{
J. Becht, A. Bath, E. Hengst, P.J. van Der Put, J. Schoonman
}

\section{To cite this version:}

J. Becht, A. Bath, E. Hengst, P.J. van Der Put, J. Schoonman. DEPOSITION OF BORON NITRIDE BY PLASMA ENHANCED CVD USING BORANE AMINE. Journal de Physique IV Proceedings, 1991, 02 (C2), pp.C2-617-C2-624. 10.1051/jp4:1991274 . jpa-00249864

\section{HAL Id: jpa-00249864 https://hal.science/jpa-00249864}

Submitted on 1 Jan 1991

HAL is a multi-disciplinary open access archive for the deposit and dissemination of scientific research documents, whether they are published or not. The documents may come from teaching and research institutions in France or abroad, or from public or private research centers.
L'archive ouverte pluridisciplinaire HAL, est destinée au dépôt et à la diffusion de documents scientifiques de niveau recherche, publiés ou non, émanant des établissements d'enseignement et de recherche français ou étrangers, des laboratoires publics ou privés. 


\title{
DEPOSITION OF BORON NITRIDE BY PLASMA ENHANCED CVD USING BORANE AMINE
}

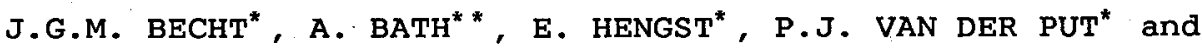 \\ J. SCHOONMAN* \\ "Laboratory for Inorganic Chemistry, Delft University of \\ Technology, Julianalaan 136. NL-2628 BL Delft, The Netherlands \\ * C.L.O.E.S, E.S.S, University of Metz, 2 rue E. Balin, \\ F-57078 Metz, France
}

\begin{abstract}
BN has been deposited with plasma enhanced CVD using borane dimethyl amine and $\mathrm{NH}$. The resulting layers are stable in air, although they contain an excess of boron, the actual amount depending on the deposition conditions. The presence of relatively large amounts of carbon in the layers indicates the incomplete decomposition of the organic fragment. Generally the layers posses the turbostratic structure. Only when a large excess of $\mathrm{NH}_{3}$ is present in the gas phase does the cubic structure develop.
\end{abstract}

\section{Introduction}

The properties of cubic BN are analogous to those of diamond: very hard, insulating, and thermodynamically stable at high pressure and temperature. Production traditionally concentrates on the formation of bulk material, which can be performed in high pressure bombs. The development of thin layers as a wear resistant coating or as an insulating film for electronic devices requires a different approach. The development of the production of diamond-like layers in a plasma environment has suggested opportunities for a similar preparation of $\mathrm{c}-\mathrm{BN} / 1 /$.

Several techniques have been employed in recent years $/ 2 /$, with a varying degree of success. Mostly the product is an amorphous material containing an excess of boron. Above $600{ }^{\circ} \mathrm{C}$ crystallization occurs, resulting in turbostratic and hexagonal $\mathrm{BN}$. Cubic $\mathrm{BN}$ is only formed if the substrate temperature is above $900^{\circ} \mathrm{C}$. Quite often $\mathrm{B} 2 \mathrm{H} 6$ has been used as a reagent, but this imposes a high risk with respect to toxicity and flammability. Less dangerous reagents can be found in the borane amines $/ 3 /$, compounds containing both boron and nitrogen. The presence of nitrogen in the molecule could facilitate the incorporation of nitrogen and possibly enhance the formation of the cubic form.

Here we report on some properties of layers prepared with borane dimethyl amine (BDMA), $\mathrm{H} 3 \mathrm{~B}-\mathrm{NH}(\mathrm{CH} 3)_{2}$, in a plasma enhanced CVD reactor, where both plasma cracking and thermolysis can occur. In order to improve the stoichiometry of the resulting layers $\mathrm{NH3}$ is added to the gas phase $/ 4 /$. 
2 Experimental

A schematic description of the reactor is given in figure 1 . The reactor itself is a glass tube. The samples are mounted on a graphite susceptor, equipped with heating rods with which the substrate temperature can be set between 200 and $700^{\circ} \mathrm{C}$. The temperature is monitored with a thermocouple. The reactor walls can be warmed in order to avoid condensation of reagents and side products. Cylindrical RF, electrodes are fitted around the reactor tube. The position of the electrodes with respect to the substrates can be changed. If hard layers are intended, the substrates are placed between the electrodes which 'also increases growth rates; for the production of insulating layers the
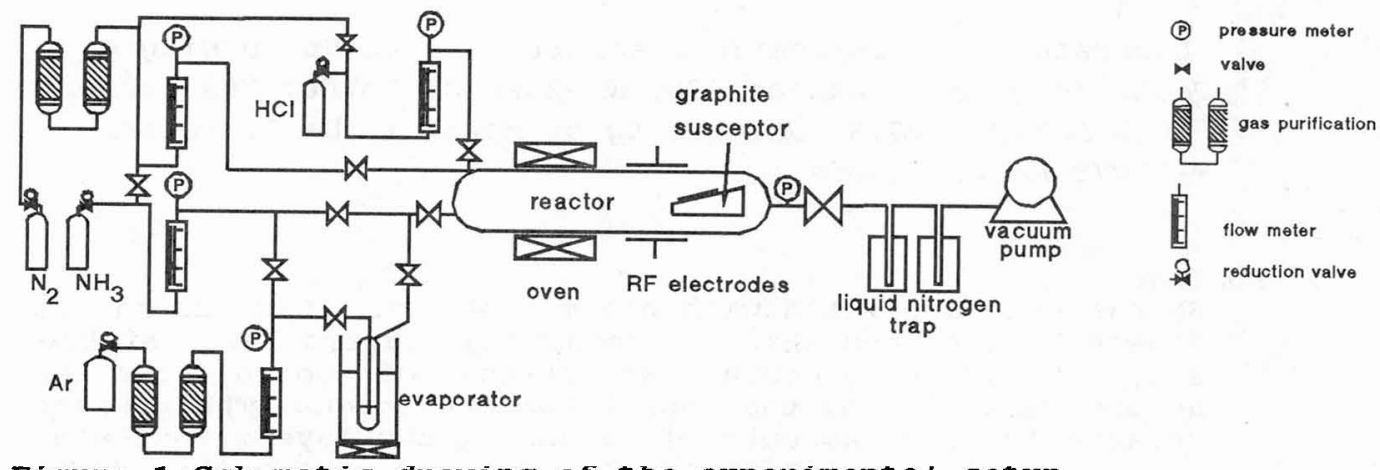

Figure 1 Schematic arawing of the experimental setup

substrates are placed downstream from the electrodes in order to reduce the incorporation of electrical defects. Prior to deposition the reactor is flushed with nitrogen (Air Products, technical purity), and occasionally the substrates are etched in situ with HCl (Air Products, purity $>99.995 \%$ ). The carrier gas argon (Air products, purity $>99.9995 \%$ ) is bubbled through the molten BDMA (Alfa Products). The temperature of the BDMA is set by a water bath at $60{ }^{\circ} \mathrm{C}$, such that polymerization of BDMA is limited (demonstrated by only a small amount of non molten white solid) while a reasonable vapor pressure is maintained (2 mbar /5/). Argon and nitrogen are dried (Chrompack filters) before use, $\mathrm{HCl}$ and $\mathrm{NH} 3$ (Air Products, purity $>99.999 \%$ ) are used as obtained. The RF plasma is created at $13.56 \mathrm{MHz}$ with a generator by Eni Power systems, ACG-5, with a maximum power of $600 \mathrm{w}$. The pressure inside the reactor is maintained with a Leybold Heraeus trivac pump, generally at 10 mbar. Residual gases are collected in a liquid nitrogen trap.

BN layers are prepared under a wide range of conditions. Process variables are: plasma power, gas pressure, reaction time, substrate temperature, argon/ammonia ratio and total flow.

Substrate materials are silicon and fused silica. Sometimes metals such as copper and platinum have been empoyed. The cleaning procedure for si consists of washing with dry acetone, and subsequent removal of the native oxide film by etching with $2 \% \mathrm{HF}$. Fused silica has only been cleaned with acetone.

Infra Red Spectroscopy (IR) and Auger Electron Spectroscopy (AES) have been used to identify the products and to determine the $B / N$ ratio in the coatings. X-ray diffraction (XRD) gives information on the crystallinity of the layers. The most direct method for measuring the resulting layer thickness is by scanning Electron Microscopy (SEM) of fractured samples. The density of a coating can be determined using the weight change of the sample after deposition. The hardness of the coatings has been determined using vickers indentation. 
The refractive index has been measured with ellipsometry at $632.8 \mathrm{~nm}$. The optical absorption in the ultraviolet-visible region has been used to determine the optical band gap.

Silicon substrates are used for IR spectroscopy. Fused silica has been used as substrate for UV-VIS analyses.

\section{Results and discussion}

\section{1 Composition and structure of the BN layers}

- $\underline{A E S}$

The composition of the layers is studied with AES. Qualitatively, the layers only contain boron and nitrogen, and traces of carbon and oxygen as shown in figure 2. The boron/nitrogen ratio of layers deposited inside the plasma is given as 2.5-3. For the layers, deposited outside the plasma a value for $B / N$ of $1.1-2.5$ has been found. The layers are stable in air and strong acids, therefore values for the boron/nitrogen ratio in the layers greater than 2 are considered to be too high $/ 6 /$. The fact that these high values are sometimes found with AES might be caused by the insulating character of $B N$, which can interfere with the quantification of the spectra. However, the IR spectra to be discussed below are also indicative of a boron excess. The oxygen levels are typically 4-5\%. The carbon levels range up to $8 \%$ for layers prepared inside the plasma and are around $20 \%$ for those deposited outside the plasma. This indicates that the decomposition of the organic part of BDMA is not complete. The sometimes yellow appearance of the transparant films agrees with this conclusion $/ 7 /$.

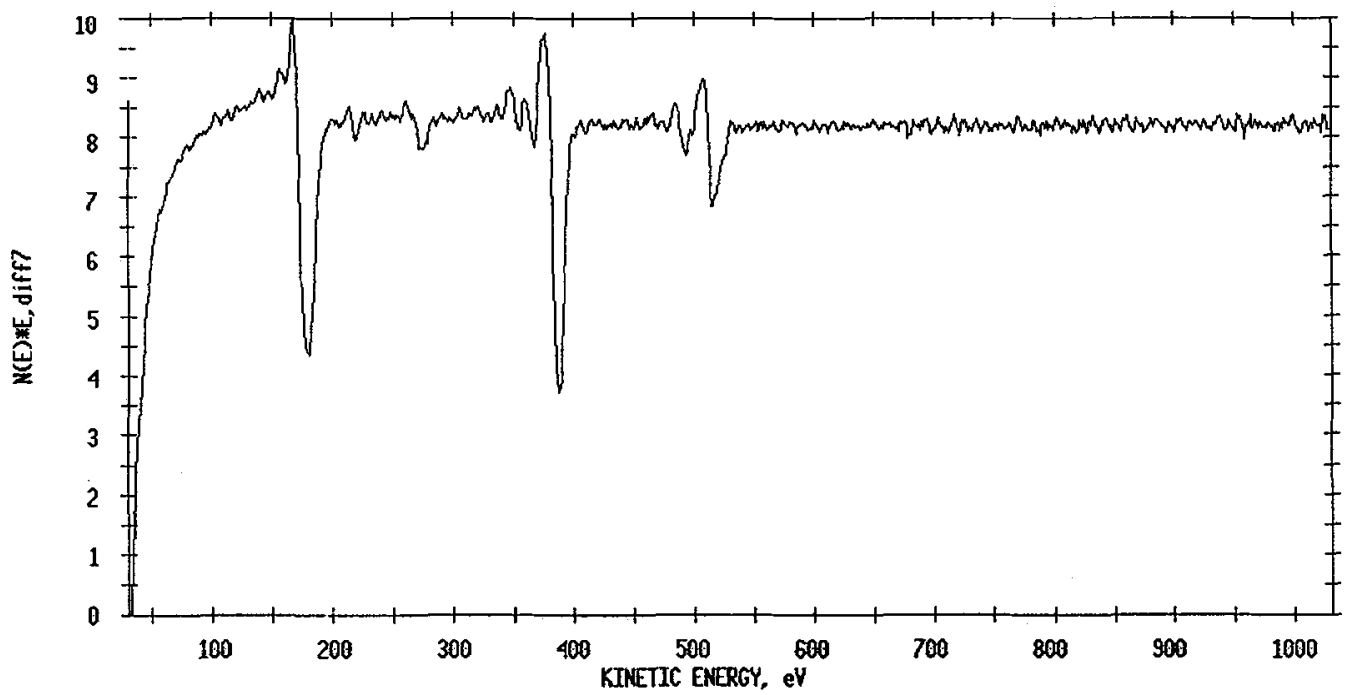

Figure 2 Typical Auger spectrum of a BN layer deposited inside the plasma.

$\underline{\text { IR }}$

Since silicon is transparent for IR radiation, it is a very suitable substrate for IR analysis. The most prominent feature of the IR absorption spectra of the BN layers, deposited both inside and outside the plasma, is the large asymmetrical band around $1380 \mathrm{~cm}^{-1}$, 
accompanied by a smaller one at about $800 \mathrm{~cm}^{-1}$ as shown in figure 3 . These two bands are characteristic for a hexagonal coordination around boron $/ 8 /$. Also a small peak around $1100 \mathrm{~cm}^{-1}$ can be distinguished, indicating a tetragonal surrounding, presumably of carbon. Between 4000 and $2000 \mathrm{~cm}^{-1}$ interference patterns are present for layers with a thickness in the range of micrometers.

sometimes $\mathrm{N}-\mathrm{H}$ bands at $3430 \mathrm{~cm}^{-1}$ are present.





Figure 3 Infrared absorptioon spectra of layers deposited at $B M D A / N H 3=0.09$, top: pressure $=1.5 \mathrm{mbar} ;$ power $=50 \mathrm{~W}$ middle: pressure $=5 \mathrm{mbar}$, power $=50 \mathrm{~W}$ bottom: pressure $=10 \mathrm{mbar}$, power $=100 \mathrm{~W}$



Figure 4-Infrared absorption spectrum of a BN layer deposited at $B D M A / N H 3=10^{-4}$, showing the cubic nature of the layer.

As is shown in figure 3 , the shape of the peaks varies along with the deposition conditions. The peak around $1380 \mathrm{~cm}^{-1}$ widens and becomes more asymmetrical. The changes resemble those attributed to the incorporation of excess boron in a hexagonal type of $\mathrm{BN} / 9 /$. The increase in absorption at 1340 and $1100 \mathrm{~cm}^{-1}$ could point to the presence of $\mathrm{B4C} / 10 /$. The assymmetry of the broad band towards higher 
wave numbers resembles that ascribed to the presence of very small particles of turbostratic $\mathrm{BN} / 11 /$.

Samples prepared at $a \mathrm{~B} / \mathrm{N}$ ratio in the gas phase of $10^{-4}$ show a sharp, isolated peak at $1100 \mathrm{~cm}^{-1}$. The band at $800 \mathrm{~cm}^{-1}$ has disappeared and the one around $1380 \mathrm{~cm}^{-1}$ has become much smaller. The $1340 \mathrm{~cm}^{-1}$ band of $\mathrm{B} 4 \mathrm{C}$ is absent. This spectrum suggests that also cubic $\mathrm{BN} / 12 /$ is formed in the presence of a large excess of ammonia.

- XRD

BN contains only very light elements. Therefore, the intensity in XRD is rather low. A low degree of crystallinity will decrease the intensity even further. Experimentally, layers thinner than $3 \mu \mathrm{m}$ do not give any pattern. Diffraction patterns for thicker layers can be characterized by three broad bands centered around $3.4 \AA, 2.1 \AA$ and $1.2 \AA$. Similar patterns have been identified as belonging to the turbostratic structure, in which layers with a planar network of hexagonal rings are stacked parallel to each other, but show random orientation and translation about the layer normal /13/. The formation of $t-B N$ by $P E-C V D$ has been described before in the literature, but at much higher substrate temperatures $/ 14 /$. At the temperatures employed here (between 300 and $700^{\circ} \mathrm{C}$ ) completely amorphous material has always been reported. The difference can be ascribed to the use of the nitrogen substituted borane.

The presence of a cubic structure in some layers, as deduced from the IR spectra, can not be corroborated with XRD, because the layers are too thin for any pattern to develop.

The presence of B4C cannot be confirmed by XRD.

\section{2 Reaction Kinetics}

The growth rate of the BN layers has been studied as a function of the deposition conditions, with the substrates inside the plasma. The growth rate is independent of the deposition time, and no nucleation effects have been observed. The growth rate is weakly dependent on the substrate temperature, and only above $600{ }^{\circ} \mathrm{C}$ does the growth rate slightly increase. This shows that cracking of the reactant molecules in the plasma is the main reaction mechanism, and that surface reaction of BDMA is only of minor importance.

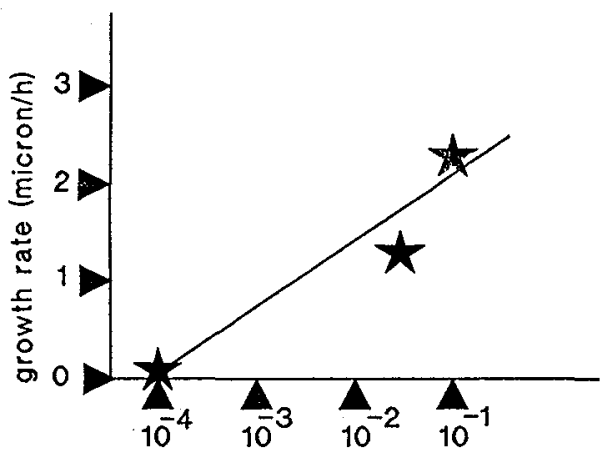

Figure 5

The relation between

the growth rate and

the ratio $B D M A / \mathrm{NH}_{3}$

$\mathrm{BDMA} / \mathrm{NH}_{3}$ ratio in gas phase

The growth rate increases with plasma power and reaction pressure, where the actual amount of BDMA in the gas phase seems to be the most important parameter, i.e. the effect of an increase of the plasma power on the growth rate is more prominent at 10 mbar, than at 1.5 
mbar. The relation between the growth rate and the ratio $\mathrm{BDMA} / \mathrm{NH} 3$ is given in figure 5. Clearly the growth rate increases dramatically if more BMDA is present in the gas phase. The total flow in the system is relatively unimportant, although at very high linear gas velocities the region with maximum deposition shifts further downstream.

If the substrates are placed outside the plasma, the growth rate is reduced significantly, presumably due to recombination of reactive species in the gas phase.

\section{3 Properties of the BN layers}

The width of the IR band around $1380 \mathrm{~cm}^{-1}$ varies with depostion conditions and is a measure of the excess of boron in the layers $/ 9 /$. The IR data can be quantified using the width of the peak (WHM) for the $1380 \mathrm{~cm}^{-1}$ band. This gives us a tool to study the relations between several properties of the BN layers. From the plot of the growth rate versus this WHM (figure 6) we learn that a higher growth rate is accompanied by a wider peak at $1380 \mathrm{~cm}^{-1}$. This implies that the higher growth rates are related to a larger incorporation of excess boron. The additional boron can better be accommodated in the less ordered turbostratic structure. The cubic form only exists in those circumstances, where the growth rate is very low and the stoichiometry is more easily preserved by the large excess of ammonia present. This is corroborated by the fact that the ordered cubic form is only found when a large surplus of $\mathrm{NH} 3$ is present in the gas phase, so that the stoichiometry is preserved more easily.

The density of the layers is not constant but gradually changes with the boron content, as is demonstrated in figure 7 . The density decreases with increasing boron excess approaching the value commonly accepted for $t-B N=1.6-2.0 \mathrm{~g} / \mathrm{cm}^{3} / 15 /$. For the layers with the narrowest peaks the density is higher, but not quite the theoretical value for $\mathrm{C}-\mathrm{BN}: 3.48 \mathrm{~g} / \mathrm{cm}^{3} / 16 /$. The scatter in the data originates from uncertainties in the determination of the layer thickness for very thin layers.

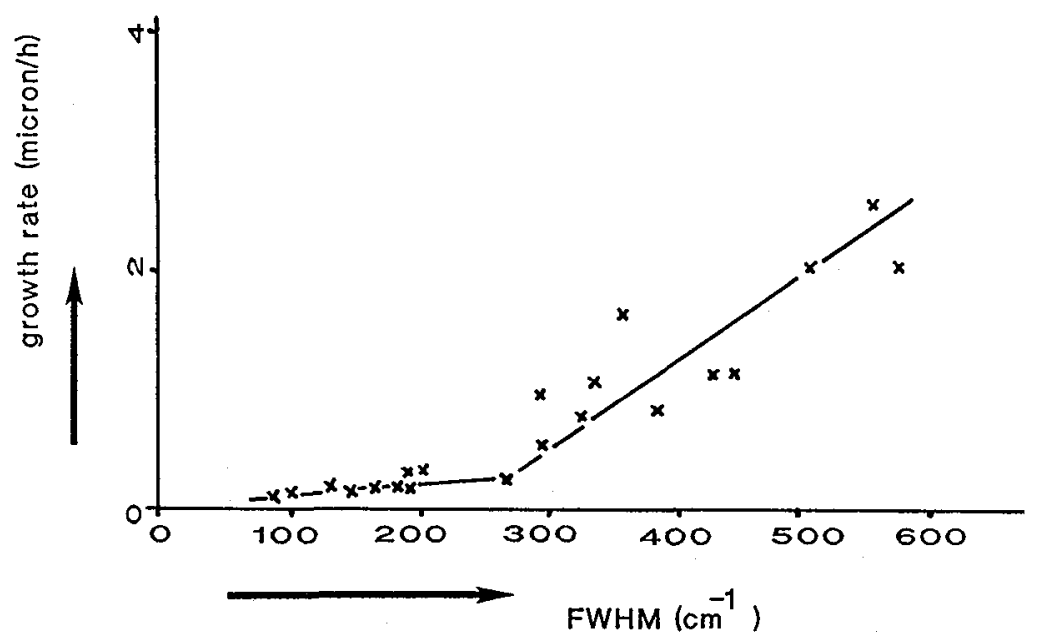

Figure 6 The relation between the growth rate of the $B N$ layers and the width of the $1380 \mathrm{~cm}^{-1}$ band of the layers in IR absorption. 
The refractive index of the layers has been measured ellipsometrically at $632.8 \mathrm{~nm}$ and with optical interference. The refractive index is generally about 1.7 , but sometimes higher values up to 2.1 have been found, indicating again a higher boron content $/ 17 /$.

For films deposited outside the plasma the optical band gap has been determined and found to be between 5.8 and $5.9 \mathrm{eV} / 18 /$ agreeing with previous work on PECVD films /19/. Excess B-B bonds narrow the optical band gap $/ 9 /$ so the experimental value indicates that although there is a certain amount of excess boron, this is not exceptionally high. The absorption spectra suggest an amorphous character of the films, in accordance with the turbostratic structure.

The adhesion of the layers is good on all the substrates, irrespective of the difference in thermal shrink. During the hardness tests brittle fracture has occurred, which indicates a good adhesion to the brittle silicon substrate. Increase of the hardness of the silicon substrates up to values ranging between 2500 and $5000 \mathrm{HV}$ have been found.



Figure 7 The relation between the density of the BN layers and the width of corresponding IR absorption spectra.

\section{Conclusions}

We have demonstrated that BDMA can successfully be used in a PECVD process to produce BN layers if additional ammonia is provided. The layers tend to contain an excess of boron, unless a large amount of ammonia is present. Some carbon is present but not in the form of B4C. Generally thicker layers possess the turbostratic structure. At a $B / N$ ratio of $10^{-4}$ in the gas phase cubic $B N$ has been

found.

Larger growth rates are related to a higher boron concentration in the layers.

Acknowledgement

This research has been financed by the EC program Euram under contract MA1E/0011/B with support of the Dutch Department of Economic Affairs in the framework of the Program for Innovative Research (IOP). 
References

/1/ Sokolowski M., Sokolowska A., Rusek A., Romanowski z., Gokieli B., Gajewska M.; J.Crystal Growth 52 (1981) 165-7

12/ Arua S.P.S., D'Amico A.; This Solid Films 157(1988) 267-282

13/ Schmolla w., Hartnagel H.L., solid state Electronics 26 (1983), 931-9

14/ Schleich D.M., Lai W.Y.F., Lam A.; Transformation of Organometallics into Common and Exotic Materials: Design and Activation; ed. R.M. Laine, Martinus Nijhoff Publ. 1988, p $178-184$

/5/ Gmelin Handbook, Supplementary Series vol 45 "Borverbindungen $14 "$, ed. K. Niedenzu, K.-C. Buschbeck; Springer Verlag Berlin 1977

/6/ Gugel E., Handbuch der Keramik, Gruppe II K2 (1975-1977), Verlag Schmidt GmbH, Freiburg i. Brg, FRG .

17/ Wiggins M.D., Aita C.R., Hickernell F.S., J.Vac.Sci.Techn.A 2 $(1984), 322$

/8/ Geick R.,' Perry C.H., Rupprecht G.; Phys.Rev. 146 (1966),543

/9/ Miyamoto M., Hirose M, Osaka Y.; Jpn.J.Appl. Phys $\underline{22}$ (1983) L216-218

/10/ Nyquist R.A., Kagel R.O., Infrared spectra of Inorganic Compounds, Academc, Nem York (1971)

/11/ Chukalin V.I., Chukanov N.V., Gurov S.V., Troitskii V.N., Filatova N.E., Rezchikova T.V., Domashneva E.P., Russ.Powder Metallurgy (1988) (1) 81-87

/12/ Gielisse P.J., Mitra S.S., Plendl J.N., Griffis R.D., Mansur L.C., Marshali R., Pascoe E.A.; Phys.Rev. 155 (1967), 1039

113/ Thomas J.Jr, Weston N.E., O'Connor T.E.; J.Amer.Chem.Soc. $84(1963) \quad 4619-4622$

/14/ Sugiyama K., Itoh H.; Materials Science Forum 54\&55 (1990) $141-152$

115/ Dugne 0., Prouhet S., Guette A., Naslain R., Fourmeaux R., Hssein K., Sevely J., Guimon C., Gonbeau D., Pfister-Guillouzo G.: J.Phys.Col1. 50(1989) (C5) 333

/16/ Lipp A., Schwertz K.A., Humold K.; J.Eur.Cer.Soc. $5(1989)$ 3-9

/17/ Murarka S.P., Chang C.C., Wang D.N.K. Smith T.E.; J.Electrochem.Soc. 126 (1979) 1951

/18/ Bath A., van der Put P.J., Becht J.G.M., Schoonman J., Lepley B.; to be published in J.Appl. Phys.

/19/ Yuzuriha T.H., Hess D.W., Thin Solid Films 140 (1986) 199 